\title{
VERIFICAÇÃO DA PRODUÇÃO DE LODO DE ETA EM FUNÇÃO DA QUANTIDADE E DA QUALIDADE DA ÁGUA
}

\author{
VERIFICATION OF THE PRODUCTION OF WTP SLUDGE AS A FUNCTION OF THE \\ QUANTITY AND QUALITY OF RAW WATER \&
}

VERIFICACIÓN DE LA PRODUCCIÓN DE LODOS ETA EN FUNCIÓN DE LA CANTIDAD Y CALIDAD DEL AGUA CRUDA \&

Recebido em: 06/04/2021 - Aprovado em: 09/09/2021 - Publicado em: 01/10/2021

doi) http://dx.doi.org/10.18011/bioeng2021v15n2p235-258

Lucas Avelino Reis dos Santos' (luucassantos.a@gmail.com)

Denise Conceição de G. Santos Michelan'1 (denise_gois@yahoo.com.br)

Taynar Mota de Jesus' (taynar.mota@gmail.com)

1 Universidade Federal de Sergipe. UFS - São Cristóvão, SERGIPE, Brasil.

\section{RESUMO}

Quantificar o lodo produzido em estações de tratamento de água (ETAs) é uma etapa fundamental para saber o quanto deste resíduo é lançado indevidamente nos mananciais e para planejar sua disposição final adequada. Este artigo aborda a comparação entre três métodos empíricos para estimativa de quantidade de lodo gerado em duas ETAs com diferente consumo de produtos químicos no tratamento de água para o município aracajuano. A pesquisa foi realizada de modo a verificar se houve diferenças nos resultados entre os anos de 2016 a 2019, comparar as estimativas de produção para cada ETA e analisar a influência das variações dos parâmetros qualitativos da água bruta e da adição de produtos químicos. Verificou-se que equações empíricas que consideram maior quantidade de variáveis são as mais apropriadas para ETAs onde o tratamento é mais complexo, enquanto que equações mais simples podem se adequar a ETAs que utilizam poucos produtos químicos.

Palavras-chave: Equações empíricas. Estação de tratamento de água. Lodo de ETA. Quantificação de lodo. 


\section{INTRODUÇÃO}

Um dos objetivos do desenvolvimento sustentável, estabelecidos pela Organização das Nações Unidas (ONU), é assegurar a disponibilidade e a gestão sustentável da água e saneamento para todos. Antes de ser distribuída, a água bruta vinda dos mananciais deve passar por diferentes etapas de tratamento na Estação de Tratamento de Água (ETA) para retirada de impurezas/resíduos. No Brasil, a água que sai da ETA deve atender aos padrões de potabilidade estabelecidos na Portaria de Consolidação $n^{\circ} 5$ do Ministério da Saúde (BRASIL, 2017). No País, do volume distribuído tratado, $75,1 \%$ recebe tratamento convencional (IBGE, 2017), no qual as duas principais fontes de resíduos advêm das unidades de decantação e filtração (LIBÂNIO, 2010).

Os resíduos gerados durante o tratamento, além de depender da composição da água bruta, dependem basicamente do tipo de processo para potabilização da água e, comumente, são: a água de lavagem dos filtros; rejeito de limpeza dos tanques de produtos químicos; e o lodo dos decantadores, o qual representa 60 a $95 \%$ do total dos resíduos gerados na ETA (REALI, 1999; RICHTER, 2009). Normalmente, este lodo é composto por hidróxidos de alumínio, carbonatos de cálcio e argilas, portanto, não é totalmente biodegradável e não se decompõe no fundo do tanque (VESILIND \& MORGAN, 2011).

Para o abastecimento de água, o uso de tecnologias com maior eficiência, como a etapa de coagulação, acarreta 0 maior consumo de produtos químicos e, consequentemente, a geração de maior volume de resíduos, constituídos por sólidos e precipitados químicos, formando o lodo de ETA (SILVA et al., 2012). Segundo Libânio (2010), as características da água bruta, o tipo e a dosagem de produtos químicos, a execução e eficiência da coagulação, além dos tipos de decantadores, são fatores que interferem nas características qualitativas e quantitativas do lodo gerado.

Além disso, a produção de resíduos em decantadores é inversamente proporcional à qualidade da água bruta em termos de cor e turbidez. De acordo com Moreira et al. (2009), o lodo formado nos decantadores de ETAs, além de apresentar a mesma composição química de sedimentos carreados pela água captada, é também acrescido de cátions utilizados no processo de coagulação.

O lodo gerado nas ETAs, apesar de conter mais de $95 \%$ em volume de água, é considerado resíduo sólido (MARGUTI et al., 2018). De acordo com a NBR 10.004 (ABNT, 2004), o lodo oriundo dos decantadores de ETA deve ser tratado e disposto de acordo com os parâmetros normatizados. Além disso, deve atender à Política Nacional de Resíduos 
Sólidos, prevista pela Lei 12.305 (BRASIL, 2010). De acordo com Marguti et al. (2018), por questões ambientais e técnicas, esses tipos de resíduos devem ser tratados antes de serem descartados no meio ambiente. Identificar novos métodos de tratamento e processos de manuseio, bem como destinos finais adequados para esses resíduos, é um desafio para engenheiros e pesquisadores em todo o mundo (SUKSIRIPATTANAPONG et al., 2015; RAMIREZ et al., 2017).

No entanto, para a maioria das ETAs brasileiras, é comum a prática de lançamento de resíduos, sem nenhum tipo de tratamento prévio, nos corpos hídricos ou em terrenos próximos às estações (MOLINA, 2010). A exemplo tem-se o lodo gerado pela ETA Poxim, que segundo Gomes Júnior et al. (2016), é disposto no corpo hídrico abastecedor da ETA, sem qualquer tratamento. Dessa mesma forma, grande parte do lodo gerado ainda é disposta ilegalmente em rios e poucas estações têm se preocupado com o tratamento e adequada disposição do resíduo. Nesse contexto, quantificar o lodo produzido em ETA constitui um dos primeiros passos para se estimar a quantidade de lodo lançado nos mananciais. É, portanto, uma etapa fundamental para o planejamento da sua disposição final, de forma prática e sustentável.

De acordo com Kawamura (1991), a melhor forma para dimensionar sistemas de tratamento de lodo, e sua quantificação, é a execução de testes em estações-piloto ou ensaios de laboratório. Porém, esses procedimentos demandam custos significativos, tornando-os menos utilizados. O balanço de massa é outra alternativa para a quantificação do lodo, na qual a produção é calculada pela relação entre massa molar dos produtos químicos aplicados e dos compostos precipitados, adicionando a concentração de sólidos na água bruta e multiplicando pela vazão (Di BERNARDO \& SABOGAL PAZ, 2008).

Existem diversas equações empíricas que estimam a produção de sólidos relacionando parâmetros como vazão afluente, dosagem de coagulante e outros produtos aplicados no tratamento, além da concentração de sólidos em suspensão na água bruta. Segundo Motta Sobrinho et al. (2019), a variabilidade de equações está associada ao tratamento que cada autor atribuiu à relação sólidos suspensos e turbidez, além da inclusão ou não do parâmetro cor. De acordo com a pesquisa realizada por Katayama et al. (2015), o cálculo de balanço de massa oferece precisão e representatividade na estimativa superiores às das fórmulas empíricas. Apesar disso, as equações são muito empregadas nos estudos devido à praticidade e principalmente por conta da falta de dados oferecidos pelas companhias operantes das estações. 
Outro fator importante é a influência da sazonalidade na produção de lodo. As equações, muitas vezes, não levam esse fator em consideração, aumentando as chances de erro na quantificação do resíduo. Variação sazonal tem maior impacto na qualidade das águas superficiais, que é a principal fonte de água bruta para ETAs (AHMAD et al., 2017). Esta variação sazonal na produção do lodo, além de depender da qualidade da água dos mananciais superficiais, sofre alteração em função da presença da chuva. Em estudo desenvolvido por Prado et al. (2021) constataram que a variação da qualidade de águas superficiais é consequência da complexa interação entre a sazonalidade chuvosa e ações antrópicas, o que resulta no comprometimento da qualidade das águas supracitadas. Isso porque, a precipitação (chuva) favorece o aporte de metais e bactérias aos mananciais superficiais.

Pesquisas desenvolvidas por Oliveira et al. (2011) apontam que partículas de solo são carreadas pelas águas de chuva, ocasionando a degradação das margens do manancial e consequentemente atuando como aporte ao manancial superficial. Para Libânio (2010) os períodos sazonais regidos por precipitação, comprometem as características das águas naturais, por meio do favorecimento de lançamento de efluentes domésticos e industriais em corpos hídricos. Portanto, quanto maior for o grau de poluição/contaminação nas águas de mananciais superficiais destinadas ao abastecimento, maior será a produção do lodo de ETA, seja por causa de maior número de impurezas que devem ser removidas da água, seja por necessitar de maior quantidade de produtos químicos para favorecer a remoção dessas impurezas da água em tratamento.

Assim, diante dos diversos caminhos existentes e viáveis para se estimar a quantificação do lodo gerado em ETA em operação, o objetivo deste trabalho foi explorar e comparar alguns dos métodos empíricos de estimativa da quantidade de lodo gerado em duas ETAs que abastecem o município de Aracaju: ETA Poxim e ETA João Ednaldo, de modo a verificar estatisticamente se estas estimativas são semelhantes, se houve variação de produção entre os anos, bem como correlação da influência da precipitação na produção do lodo.

\section{MATERIAIS E MÉTODOS}

\section{1 ÁREAS DE ESTUDO}

Neste trabalho, foram estudadas duas das três principais ETAs, em termos de medida de vazão, que abastecem Aracaju-SE: ETA Poxim e ETA João Ednaldo, que 
captam água dos rios Poxim e São Francisco, respectivamente, durante o período de 2016 a 2019. Ambas são operadas pela Companhia de Saneamento do Estado de Sergipe DESO.

A ETA Poxim localiza-se no bairro Capucho, em Aracaju-SE, e capta água do rio Poxim, que contribui para o abastecimento de aproximadamente $30 \%$ da capital sergipana (ROCHA et al., 2014). Segundo a DESO (2020), a população é atendida pela captação desse rio ao longo de $8,5 \mathrm{~km}$ de adutora e produção de água tratada de $3.000 \mathrm{~m}^{3} \cdot \mathrm{h}^{-1}$. Segundo Farias (2011), o curso d'água bruta está sujeito a diversos pontos de lançamento de esgoto clandestinos devido à falta de saneamento adequado das localidades urbanas presentes em torno do rio. Este fator contribui para o comprometimento da qualidade de água bruta direcionada para a ETA.

O tipo de tratamento adotado na ETA Poxim é de ciclo completo (convencional), composto pelas etapas de mistura rápida com coagulação, floculação, decantação, filtração, fluoretação e desinfecção. Inicialmente, a água bruta recebe a adição de cal hidratada para correção de $\mathrm{pH}$. A estação também adota uma etapa pré-oxidação com permanganato de potássio, a fim de oxidar matéria orgânica e remover cor, evitando a formação de subprodutos.

A coagulação ocorre na calha Parshall, por meio de mistura rápida hidráulica, onde é adicionado sulfato de alumínio e, dependendo da qualidade da água bruta, é aplicado o adsorvente carvão ativado em pó para remoção de causadores de mau odor.

Após passar pela calha, a água segue para dois floculadores hidráulicos de fluxo vertical, onde é aplicado polímero aniônico para auxiliar na formação dos flocos. Em seguida, a água segue para os quatro decantadores retangulares convencionais. O lodo sedimentado permanece no fundo do decantador (maior volume) e a água segue para os filtros, juntamente com os flocos que não alcançaram peso suficiente para sedimentar no decantador. A filtração de fluxo descendente é feita em seis filtros rápidos, seguida pela adição de ácido fluossilícico em câmara de contato. Parte da desinfecção por meio de cloro gasoso ocorre no canal de água após decantação e parte no tanque de contato. Em seguida, a água é destinada ao reservatório da estação, pronta para a distribuição.

A ETA João Ednaldo fica localizada em Nossa Senhora do Socorro-SE, próxima à cidade de Aracaju, no bairro Sobrado. Também adota o tratamento por ciclo completo e opera com dois módulos, um antigo (construído em 1980) e outro mais novo implantado 
após ampliação, em 2017. Segundo a DESO (2020), a ETA atende à população ao longo de $188 \mathrm{~km}$ de adutora e tem produção de água tratada de $6.773 .940 \mathrm{~m}^{3} \cdot \mathrm{h}^{-1}$.

A maior parte da água captada provém do rio São Francisco, através de adutora em concreto localizada $2 \mathrm{~km}$ a montante da cidade de Propriá-SE. Eventualmente, a estação recebe também água do rio Poxim, devido ao aumento da demanda de água tratada. $\mathrm{A}$ vazão em cada módulo não é medida, uma vez que não há medidor instalado antes da divisão de vazões para os módulos, podendo um módulo ou outro receber mais que a metade da vazão total. Dessa forma, não se leva em consideração a diferença da qualidade da água proveniente de cada módulo. A captação da água do rio Poxim para a ETA João Ednaldo é feita em um ponto denominado Poxim II, no Bairro Capucho, em Aracaju.

Ocasionalmente, a água bruta recebe a adição de cal hidratada para correção de $\mathrm{pH}$, logo em seguida passa pela única calha Parshall da ETA João Ednaldo, onde ocorre a etapa de coagulação por mistura rápida hidráulica, com a adição de sulfato de alumínio. É utilizado também, eventualmente, polímero aniônico para auxiliar na floculação.

No módulo antigo, a água passa por dois floculadores hidráulicos verticais com chicanas. Em seguida, passa por quatro decantadores com placas defletoras paralelas e descarga de fundo para retirada do lodo, por meio de canal longitudinal no fundo do tanque. $\mathrm{Na}$ sequência, a água segue para seis filtros rápidos descendentes com camada de antracito apoiada na camada de areia.

No módulo novo, a água passa por quatro unidades de floculadores hidráulicos verticais sem chicanas. Em seguida, passa pelos quatro decantadores do tipo convencional com calhas coletoras em paralelo e depois por três filtros rápidos descendentes.

Após a água sair dos filtros, segue para o reservatório, independente da procedência do tratamento (módulo antigo ou novo) e assim recebe o cloro gasoso como produto de desinfecção e o ácido fluossilícico para a fluoretação da água.

\subsection{EQUAÇÕES EMPÍRICAS PARA A QUANTIFICAÇÃO DA PRODUÇÃO DO LODO DE ETA}

Há diversas equações disponíveis na literatura para a estimativa da produção de lodo. Entre as quais estão as propostas pela American Water Work Association - AWWA (1999), pela Association Francaise Pour L'etude Des Eaux - AFEE, por Kawamura (1991), por Richter (2009) e por Cornwell (1987). 
Assim, neste estudo, a quantificação do lodo gerado nos decantadores das ETAs foi estimada utilizando as equações propostas por AWWA (1999), Richter (2009) e Cornwell et al. (1987) por serem as mais usuais, conforme a pesquisa de literatura, além de que algumas outras correspondem às ramificações e adaptações destas supracitadas. Os modelos foram adotados, também, devido à melhor viabilidade para aplicação dos dados de entrada, a exemplo de vazão, dosagem de coagulantes, turbidez e cor da água bruta.

Assim, AWWA (1999) propõe a Equação 1 que, empiricamente, estima a produção do lodo para ETAs de ciclo completo.

$$
W=86,4 \times Q \times\left(4,89 D+S S T+D_{P}+D_{c a p}+0,1 \times D_{c a l}\right) \times 10^{-3}
$$

onde:

W $\rightarrow$ Produção de SST (kg.dia-1 ); Q $\rightarrow$ Vazão de água bruta a ser tratada (L.S $\left.{ }^{-1}\right) ; D \rightarrow$ Dosagem de sulfato de alumínio $\left(\mathrm{mgAl}^{-1}{ }^{-1}\right)$; SST $\rightarrow$ Sólidos em suspensão na água bruta $\left(\mathrm{mgSST} . \mathrm{L}^{-1}\right) ; \mathrm{D}_{\mathrm{p}} \rightarrow$ Dosagem de polímero seco $\left(\mathrm{mg} . \mathrm{L}^{-1}\right) ; \mathrm{D}_{\text {cap }} \rightarrow$ Dosagem de dureza cálcica removida $\left(\mathrm{mg}_{\mathrm{L}} \mathrm{L}^{-1} \mathrm{CaCO}_{3}\right) ; \mathrm{D}_{\text {cal }} \rightarrow$ Dosagem de cal $\left(\mathrm{mg} \cdot \mathrm{L}^{-1}\right)$.

A Equação 2, proposta por Richter (2009), considera valores de cor e de turbidez, além da dosagem de coagulante, para estimar a quantidade de lodo produzido na ETA.

$$
S=\left(0,2 \times C+k_{1} \times T+k_{2} \times D\right) \times 10^{-3}
$$

na qual:

$\mathrm{S} \rightarrow$ Massa de sólidos secos precipitada $\left(\mathrm{kg} \cdot \mathrm{m}^{-3}\right.$ de água tratada); $\mathrm{C} \rightarrow \mathrm{Cor}$ da água bruta $(\mathrm{uH}) ; \mathrm{T} \rightarrow$ Turbidez da água bruta (UNT); D $\rightarrow$ Dosagem de coagulante $\left(\mathrm{mg}^{\mathrm{L}} \mathrm{L}^{-1}\right) ; \mathrm{k}_{1} \rightarrow$ Coeficiente de relação entre sólidos suspensos totais e turbidez, adotado 1,3, conforme indicado pelo autor; $\mathrm{k}_{2} \rightarrow$ Relação estequiométrica na formação do precipitado de hidróxido que depende do coagulante utilizado, adotado 0,26.

Com o valor de S, encontrado na Equação 2, a massa de sólidos por unidade de tempo (Ms), em $\mathrm{kg} \cdot \mathrm{dia}^{-1}$, removida na unidade de processo pode ser calculada pela Equação 3.

$$
M_{S}=\eta \times S \times Q
$$

em que: 
$\eta \rightarrow$ Rendimento da unidade de processo, adotado $100 \%$, conforme indicado pelo autor; $\mathrm{S} \rightarrow$ Massa de sólidos secos precipitada $\left(\mathrm{kg} \cdot \mathrm{m}^{-3}\right.$ de água tratada); $\mathrm{Q} \rightarrow$ Vazão (m³.dia$1)$.

Para Cornwell et al. (1987), citado por Barroso e Cordeiro (2002), as reações do coagulante e de outros produtos químicos, além da quantidade de sólidos suspensos totais, são consideradas na relação empírica para explicar suas contribuições para formação do lodo de ETA, que é expressa pela Equação 4.

$$
S=Q \times(4,89 \times A l+S S T+A)
$$

onde:

$\mathrm{S} \rightarrow$ Produção de sólidos $\left(\mathrm{kg} \cdot \mathrm{dia}^{-1}\right) ; \mathrm{Q} \rightarrow$ Vazão de água bruta $\left(\mathrm{m}^{3} \cdot \mathrm{dia}^{-1}\right) ; \mathrm{Al} \rightarrow$ Dosagem de sulfato de alumínio, expresso como $\mathrm{Al}\left(\mathrm{mg} . \mathrm{L}^{-1}\right)$; SST $\rightarrow$ Concentração de sólidos suspensos na água bruta $\left(\mathrm{mg} \cdot \mathrm{L}^{-1}\right) ; \mathrm{A} \rightarrow$ Dosagem de produtos químicos adicionados, tais como polímeros, carvão ativado etc $\left(m g \cdot \mathrm{L}^{-1}\right)$.

Para a quantidade de sólidos suspensos, solicitadas nas Equações 1 e 4, foi utilizada a relação entre a turbidez e unidade de SST expressa na Equação 5.

$$
\text { SST }\left(m g \cdot L^{-1}\right)=b \times \text { Turbidez }(\text { UNT) }
$$

O valor de b (coeficiente de turbidez) varia entre 1 e 2 (HOWE et al., 2017). Nesse estudo, foi utilizado o valor de 1,5 para todos os cálculos.

\subsection{DADOS DO ESTUDO}

Foram analisadas séries históricas dos produtos químicos sulfato de alumínio, permanganato de potássio, cal hidratada, carvão ativado, polímero aniônico, da dureza cálcica e da vazão das ETAs, abrangendo o período de 2016 a 2019, a fim de avaliar comparativamente a aplicação desses parâmetros em fórmulas empíricas para quantificação de lodo. A partir destes dados, foram calculados valores médios diários para inserção nas equações apresentadas.

As séries temporais analisadas correspondentes ao período supracitado, apresentaram informações mensais referentes à água bruta captada nos mananciais para as ETAs em estudo. Os dados foram extraídos de boletins de análise elaborados pela 
gerência de controle e vigilância da qualidade da água das estações de tratamento, em que são apresentados os parâmetros físico-químicos mensais.

\subsection{DADOS DO ESTUDO}

Os dados foram analisados mensalmente para as duas estações e, para cada, foram feitas análises estatísticas de frequência para expressar as variações mensais da produção de lodo. A partir desses valores apresentados, obtiveram-se valores médios anuais, medianas, mínimos, máximos e seus desvios padrões. Os resultados obtidos foram agrupados e posteriormente tratados através do software IBM SPSS Statistics 20.

Com os valores médios anuais de produção de lodo calculados, foi feito o tratamento estatístico dos dados apresentados nas três fórmulas. Foi realizada análise de variância de uma via (ANOVA-One Way) com o objetivo de avaliar se haviam diferenças nos níveis de produção de lodo entre os diferentes anos para cada equação proposta.

A normalidade dos dados foi avaliada por meio dos testes Kolmogorov-Smirnov e Shapiro-Wilk. O pressuposto de homogeneidade de variância foi avaliado por meio do teste de Levene. Ademais, foram realizados procedimentos de bootstrapping (1000 reamostragens; 95\% IC BCa) para se obter maior confiabilidade dos resultados e corrigir desvios de normalidade da distribuição da amostra, além de apresentar um intervalo de confiança de 95\% para as diferenças entre as médias (HAUKOOS \& LEWIS, 2005). A avaliação de correlação cronológica foi feita por meio da técnica Tukey, utilizada para encontrar quais são os grupos diferentes.

A ANOVA, juntamente com o Tukey, também foi aplicada para efeito de comparação entre os resultados das três equações, a fim de verificar se há diferença significativa entre essas, com intervalo de confiança de $95 \%$ e, enfim, mostrar quais retornam dados estatisticamente diferentes. Considerando a heterogeneidade de variância, foi solicitada a correção de Welch.

Foi realizado um teste de correlação para analisar a influência da precipitação na produção de lodo. Dessa forma, os valores de coeficientes foram interpretados de acordo com a convenção proposta por Callegari-Jacques (2003) apud Baba et al. (2014), que consideram correlações de $0 \%$ a $19 \%$ como muito fracas, de $20 \%$ a $39 \%$ como fracas, entre $40 \%$ e $69 \%$ como moderadas, entre $70 \%$ e $89 \%$ como fortes e entre $90 \%$ e $100 \%$ como muito fortes. 


\section{RESULTADOS E DISCUSSÃO}

Com base no que foi descrito quanto aos produtos químicos utilizados seja na ETA Poxim quanto na ETA João Ednaldo, observa-se que a qualidade da água bruta do rio Poxim, que abastece a ETA Poxim, necessita de maior número de tipos de produtos para realizar o tratamento convencional, se comparado com a variedade de tipos de produtos químicos utilizados na ETA João Ednaldo, que recebe água proveniente do rio São Francisco e eventualmente do rio Poxim. Diante disso, os resultados apresentados para as ETAs quanto às estimativas de produção de lodo são influenciados pelos produtos químicos.

A produção diária de lodo foi calculada através das Equações 1, 3 e 4. Os resultados foram compactados na forma de médias, para cada um dos quatro anos analisados, assim como os valores de mediana, desvio padrão, mínimo e máximo, apresentados nas Tabelas 1, 2 e 3, para a equação de AWWA (1999), Richter (2009) e Cornwell et al. (1987), respectivamente, para a ETA Poxim.

Tabela 1 - Produção de lodo (ton.dia-1) por AWWA (1999) - ETA Poxim.

\begin{tabular}{ccccc}
\hline Estatística descritiva & $\mathbf{2 0 1 6}$ & $\mathbf{2 0 1 7}$ & $\mathbf{2 0 1 8}$ & $\mathbf{2 0 1 9}$ \\
\hline Média & 184,80 & 183,84 & 194,95 & 147,01 \\
Mediana & 180,85 & 181,36 & 197,71 & 141,33 \\
Desvio Padrão & 29,31 & 28,85 & 17,17 & 44,25 \\
Mínimo & 136,30 & 144,95 & 156,39 & 69,69 \\
Máximo & 230,10 & 216,56 & 227,12 & 229,90 \\
\hline
\end{tabular}

Fonte: Autores (2021).

Tabela 2 - Produção de lodo (ton.dia-1) por Richter (2009) - ETA Poxim.

\begin{tabular}{ccccc}
\hline Estatística descritiva & $\mathbf{2 0 1 6}$ & $\mathbf{2 0 1 7}$ & $\mathbf{2 0 1 8}$ & $\mathbf{2 0 1 9}$ \\
\hline Média & 60,56 & 63,85 & 37,00 & 45,44 \\
Mediana & 50,01 & 61,90 & 35,94 & 40,88 \\
Desvio Padrão & 35,89 & 25,03 & 5,61 & 33,29 \\
Mínimo & 26,98 & 33,78 & 30,07 & 6,28 \\
Máximo & 147,05 & 108,68 & 49,58 & 132,96 \\
\hline
\end{tabular}

Fonte: Autores (2021).

Tabela 3 - Produção de lodo (ton.dia-1) por Cornwell (1987) - ETA Poxim.

\begin{tabular}{ccccc}
\hline Estatística descritiva & $\mathbf{2 0 1 6}$ & $\mathbf{2 0 1 7}$ & $\mathbf{2 0 1 8}$ & $\mathbf{2 0 1 9}$ \\
\hline Média & 83,42 & 107,92 & 88,66 & 77,99 \\
Mediana & 76,07 & 106,37 & 86,12 & 69,13 \\
Desvio Padrão & 35,96 & 28,99 & 13,24 & 26,51 \\
Mínimo & 46,65 & 66,66 & 73,06 & 55,57 \\
Máximo & 162,69 & 166,35 & 117,45 & 134,44 \\
\hline
\end{tabular}

Fonte: Autores (2021). 
As Tabelas 4, 5 e 6 apresentam os resultados descritivos da produção diária de lodo para a ETA João Ednaldo (JE), para as equações de AWWA (1999), Richter (2009) e Cornwell et al. (1987), respectivamente.

Tabela 4 - Estatística descritiva da geração diária de lodo (ton.dia-1) por AWWA (1999) - ETA João

\begin{tabular}{|c|c|c|c|c|}
\hline \multicolumn{5}{|c|}{ Ednaldo. } \\
\hline Estatística descritiva & 2016 & 2017 & 2018 & 2019 \\
\hline Média & 149,76 & 185,13 & 201,34 & 190,54 \\
\hline Mediana & 138,49 & 169,49 & 130,36 & 177,04 \\
\hline Desvio Padrão & 43,06 & 79,41 & 225,72 & 64,53 \\
\hline Mínimo & 108,73 & 115,34 & 109,57 & 135,65 \\
\hline Máximo & 259,06 & 409,46 & 912,81 & 363,05 \\
\hline
\end{tabular}

Fonte: Autores (2021).

Tabela 5 - Estatística descritiva da geração diária de lodo (ton.dia-1) por Richter (2009) - ETA João Ednaldo.

\begin{tabular}{ccccc}
\hline Estatística descritiva & $\mathbf{2 0 1 6}$ & $\mathbf{2 0 1 7}$ & $\mathbf{2 0 1 8}$ & $\mathbf{2 0 1 9}$ \\
\hline Média & 28,94 & 36,59 & 30,40 & 48,50 \\
Mediana & 22,95 & 23,52 & 10,29 & 23,04 \\
Desvio Padrão & 20,32 & 44,99 & 43,61 & 64,62 \\
Mínimo & 7,11 & 6,34 & 7,30 & 3,78 \\
Máximo & 65,45 & 168,89 & 159,63 & 235,76 \\
\hline
\end{tabular}

Fonte: Autores (2021).

Tabela 6 - Estatística descritiva da geração diária de lodo (ton.dia-1 ${ }^{-1}$ por Cornwell (1987) - ETA João Ednaldo.

\begin{tabular}{ccccc}
\hline Estatística descritiva & $\mathbf{2 0 1 6}$ & $\mathbf{2 0 1 7}$ & $\mathbf{2 0 1 8}$ & $\mathbf{2 0 1 9}$ \\
\hline Média & 33,64 & 58,13 & 36,84 & 58,40 \\
Mediana & 27,95 & 46,01 & 28,87 & 39,41 \\
Desvio Padrão & 18,92 & 58,27 & 27,03 & 58,94 \\
Mínimo & 13,14 & 11,23 & 10,91 & 13,76 \\
Máximo & 62,89 & 220,61 & 107,95 & 224,08 \\
\hline
\end{tabular}

Fonte: Autores (2021).

Em observação aos desvios padrão apresentados nas Tabelas 1 a 6 , os mesmos indicam o quanto a amostragem encontra-se próxima ou dispersa entre os dados estudados. Associando essa informação à quantidade de variáveis integrantes da Equação proposta por AWWA (1999), nota-se que essa combinação pode ocasionar desvios padrão significativo, a exemplo de 225,72 ton.dia-1, ilustrado na Tabela 4. Ao passo que, amostragens com dados próximos da média, aplicados em equações com número menor de variáveis, tende a desvios padrão menos significativos, a exemplo da aplicação de dados na Equação proposta por Cornwell (1987). 
3.1 PRODUÇÃO DE LODO POR VAZÃO DE ÁGUA TRATADA: ETA POXIM E ETA JOÃO EDNALDO

As Tabelas 7 e 8 apresentam os resultados descritivos da produção de lodo em g.m

3 para as ETA Poxim e João Ednaldo, respectivamente, no ano de 2019.

A ETA João Ednaldo trabalha com vazão muito maior de água captada, se comparada com a ETA Poxim, e, apesar disso, a produção de lodo média por vazão da água bruta, calculada para as equações da AWWA, Richter e Cornwell é, respectivamente, cerca de $160 \%, 210 \%$ e 350\% menor em relação a da ETA Poxim. Esses valores mais significativos da ETA Poxim podem ser justificados pela maior quantidade de produtos químicos adicionados durante o tratamento devido à influência da qualidade da água.

A qualidade da água bruta obtida, nesse estudo, corrobora o que Batista et al. (2019) encontraram ao analisar a água bruta em termos de turbidez e cor para a água do rio São Francisco que abastece a ETA João Ednaldo e para o rio Poxim, que em algumas épocas do ano, contribui com abastecimento de água a ETA João Ednaldo.

Tabela 7- Produção de lodo (g.m-3) em 2019 - ETA Poxim.

\begin{tabular}{cccc}
\hline Estatística descritiva & AWWA & RICHTER & CORNWELL \\
\hline Média & 125,77 & 37,99 & 66,38 \\
Mediana & 127,55 & 35,54 & 59,76 \\
Desvio Padrão & 33,01 & 24,43 & 18,32 \\
Mínimo & 60,24 & 5,43 & 48,04 \\
Máximo & 190,71 & 96,92 & 99,31 \\
\hline
\end{tabular}

Fonte: Autores (2021).

Tabela 8 - Produção de lodo (g.m-3) em 2019 - ETA João Ednaldo

\begin{tabular}{cccc}
\hline Estatística descritiva & AWWA & RICHTER & CORNWELL \\
\hline Média & 47,95 & 12,24 & 14,78 \\
Mediana & 41,26 & 5,58 & 9,55 \\
Desvio Padrão & 16,06 & 15,82 & 14,53 \\
Mínimo & 34,28 & 0,87 & 3,23 \\
Máximo & 87,88 & 57,07 & 54,24 \\
\hline \multicolumn{2}{r}{ Fonte: Autores $(2021)}$.
\end{tabular}

\subsection{ANÁLISE ESTATÍSTICA}

Na ETA Poxim, os testes de distribuição de normalidade, Kolmogorov-Smirnov e Shapiro-Wilk, demonstraram que apenas os resultados das equações propostas pela AWWA (1999) e por Cornwell (1987) apresentaram distribuição normal, com p > 0,05 (hipótese para distribuição normal). Na ETA João Ednaldo, os testes mostraram que os valores de produção diária de lodo nos anos estudados não apresentaram distribuição 
Verificação da produção de lodo de eta em função da...

normal, com valores de $\mathrm{p}<0,05$ para os resultados das três equações em estudo. Entretanto o teste de Levene demonstrou que os grupos (anos) apresentam homogeneidade de variância, apresentando valores de $p>0,05$ (hipótese para distribuição homogênea).

Em ambas as ETAs, os resultados da ANOVA e do teste de Tukey, interpretados por meio de procedimentos de bootstrapping, demonstraram que não foram encontradas diferenças significativas (Sig.) entre os anos, apresentaram valores de $p>0,05$, ilustrados nas Tabelas 9 e 10, para ETA Poxim e João Ednaldo, respectivamente.

Tabela 9 - Teste Tukey ETA Poxim.

\begin{tabular}{cccccc}
\hline $\begin{array}{c}\text { Equações } \\
\text { proposta por: }\end{array}$ & $\mathbf{2 0 1 6}$ & $\mathbf{2 0 1 7}$ & $\mathbf{2 0 1 9}$ & $\mathbf{2 0 1 8}$ & Sig. \\
\hline AWWA & 147,01 & 183,84 & 184,80 & 194,95 & p \\
Richter & 37,00 & 45,44 & 60,56 & 63,85 & 0,096 \\
Cornwell & 77,99 & 83,42 & 88,66 & 107,92 & 0,143 \\
\hline
\end{tabular}

São exibidas as médias para os grupos em subconjuntos homogêneos.

Sig. $\rightarrow$ diferenças significativas

$p \rightarrow$ homogeneidade de variância

Fonte: Autores (2021).

Tabela 10 - Teste Tukey ETA João Ednaldo.

\begin{tabular}{cccccc}
\hline $\begin{array}{c}\text { Equações } \\
\text { proposta por: }\end{array}$ & $\mathbf{2 0 1 6}$ & $\mathbf{2 0 1 7}$ & $\mathbf{2 0 1 9}$ & $\mathbf{2 0 1 8}$ & $\begin{array}{c}\text { Sig. } \\
\text { p }\end{array}$ \\
\hline AWWA & 149,76 & 185,13 & 190,54 & 201,34 & 0,748 \\
Richter & 28,94 & 30,40 & 36,59 & 48,50 & 0,728 \\
Cornwell & 33,64 & 36,84 & 58,13 & 58,40 & 0,531 \\
\hline
\end{tabular}

São exibidas as médias para os grupos em subconjuntos homogêneos.

Sig. $\rightarrow$ diferenças significativas

$\mathrm{p} \rightarrow$ homogeneidade de variância

Fonte: Autores (2021).

Analisando os valores médios mensais para a ETA Poxim durante o período de estudo, os resultados da ANOVA demonstraram que haviam diferenças entre as estimativas das três fórmulas [Welch's $F(2,93,25)=182,7, p<0,001$ ]. $O$ teste de Tukey apresentou diferenças significativas entre todas as equações, com valores de $p<0,05$ para todas as comparações, apresentados na Tabela 11.

Após a avaliação ANOVA [Welch's $F(2,86,83)=29,55, p<0,001$ ], o teste de Tukey para a ETA João Ednaldo demonstrou que foram encontradas diferenças significativas entre a fórmula da AWWA (1999) e a de Richter (2009) e entre a da AWWA (1999) e a de 
Cornwell (1987), com $p<0,05$, e similaridade entre a de Richter e a de Cornwell, com $p>$ 0,05, para os anos 2016-2019. Os valores estão apresentados na Tabela 12.

A diferença entre os resultados das fórmulas acontece devido à influência das variáveis que são consideradas em cada equação empírica. As diferenças significativas podem ocorrer em uma estação e não ocorrer em outra, devido às distinções entre os parâmetros físico-químicos e ao consumo de produtos em cada ETA.

Tabela 11 - Teste Tukey ETA Poxim.

\begin{tabular}{cccc}
\hline & AWWA x Richter & AWWA x Cornwell & Richter x Cornwell \\
\cline { 2 - 4 } $\mathrm{p}$ & $<0,001$ & $<0,001$ & $<0,001$ \\
\hline
\end{tabular}

Fonte: Autores (2021).

Tabela 12 - Teste Tukey ETA João Ednaldo.

\begin{tabular}{cccc}
\hline & AWWA x Richter & AWWA x Cornwell & Richter $x$ Cornwell \\
\cline { 2 - 4 } & $<0,001$ & $<0,001$ & 0,792 \\
\hline
\end{tabular}

Fonte: Autores (2021).

\subsection{INFLUÊNCIA DOS PARÂMETROS}

A influência dos parâmetros na produção de lodo, para a Equação da AWWA, de Richter e de Cornwell, pode ser melhor visualizada na Figura 1, onde são ilustradas as participações das variáveis de acordo com a média diária para cada mês na ETA Poxim. $\mathrm{O}$ estudo estatístico mostrou que não houve diferença significativa de produção de lodo entre os anos estudados, portanto optou-se por fazer esta análise apenas para o ano de 2019, com as Equações 1, 3 e 4, devido ao grande volume de dados.

Foi possível notar, através dos gráficos, que a variação da produção de lodo acompanha a variação dos valores de sólidos suspensos totais existentes na água bruta, seguida pela quantidade do coagulante e dos outros produtos químicos aplicados durante o processo de tratamento.

As equações sugeridas pela AWWA (1999) e por Cornwell et al. (1987) apud Barroso e Cordeiro (2002) consideram características físico-químicas e parâmetros como dosagem de coagulante e produtos químicos adicionais. Porém, além disso, a primeira considera também a dureza cálcica da água bruta, fazendo com que haja diferenças quando comparadas entre si. A equação da AWWA (1999) apresentou um pico de produção de lodo em maio devido ao maior valor do parâmetro dureza cálcica. 
Figura 1: Parâmetros para estimativa de produção de lodo na ETA Poxim.

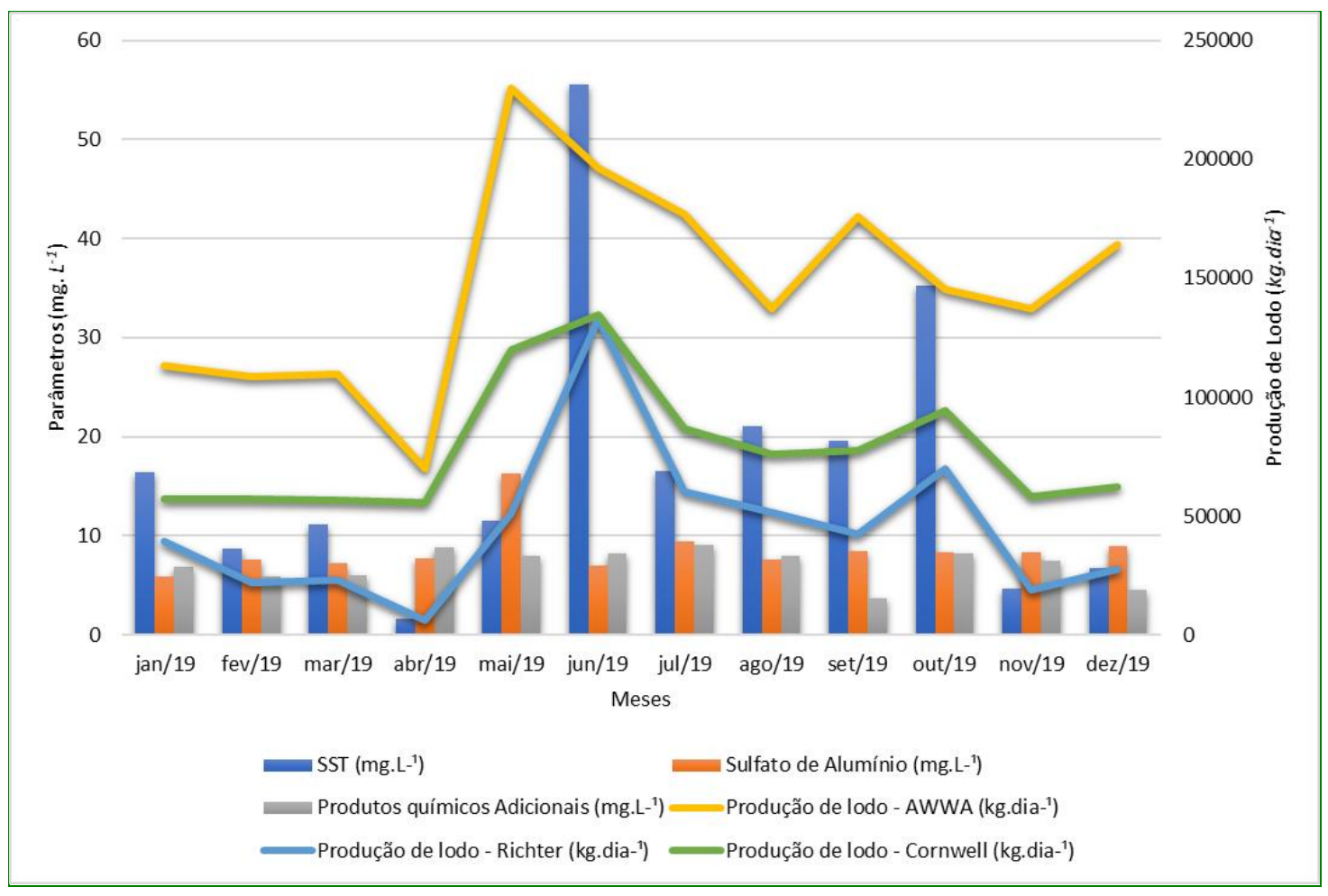

Fonte: Autores (2021).

A equação proposta por Richter (2001) admite a produção de lodo diretamente relacionada com a turbidez, cor e a dosagem de coagulante, subestimando a produção de lodo por desconsiderar parâmetros como produtos químicos adicionais e, portanto, suas estimativas de produção se diferem das outras duas.

Na Figura 1, observa-se maior quantidade de lodo formada nos meses de junho a outubro. Um estudo realizado por Aragão et al. (2013) mostrou que o período chuvoso na capital sergipana é de abril a agosto, com máximas de pluviosidade se concentrando em maio, junho e julho. Assim, maiores valores de precipitação podem justificar os picos de produção de lodo estimados pelas equações nesses meses, devido aos maiores valores de turbidez. Esse comportamento ocorre devido ao carreamento de partículas para os corpos d'água pela chuva, que ocasiona o aumento da presença de sólidos na água e, como consequência direta, o aumento de turbidez. Nas equações, os valores significativos de turbidez da água bruta causam o aumento dos valores de sólidos suspensos totais calculados pela Equação 5.

A Figura 2 mostra a influência dos parâmetros para a produção de lodo na ETA João Ednaldo durante o ano de 2019, para as equações da AWWA, de Richter e de Cornwell, respectivamente. 
Figura 2: Parâmetros para estimativa de produção de lodo na ETA João Ednaldo.

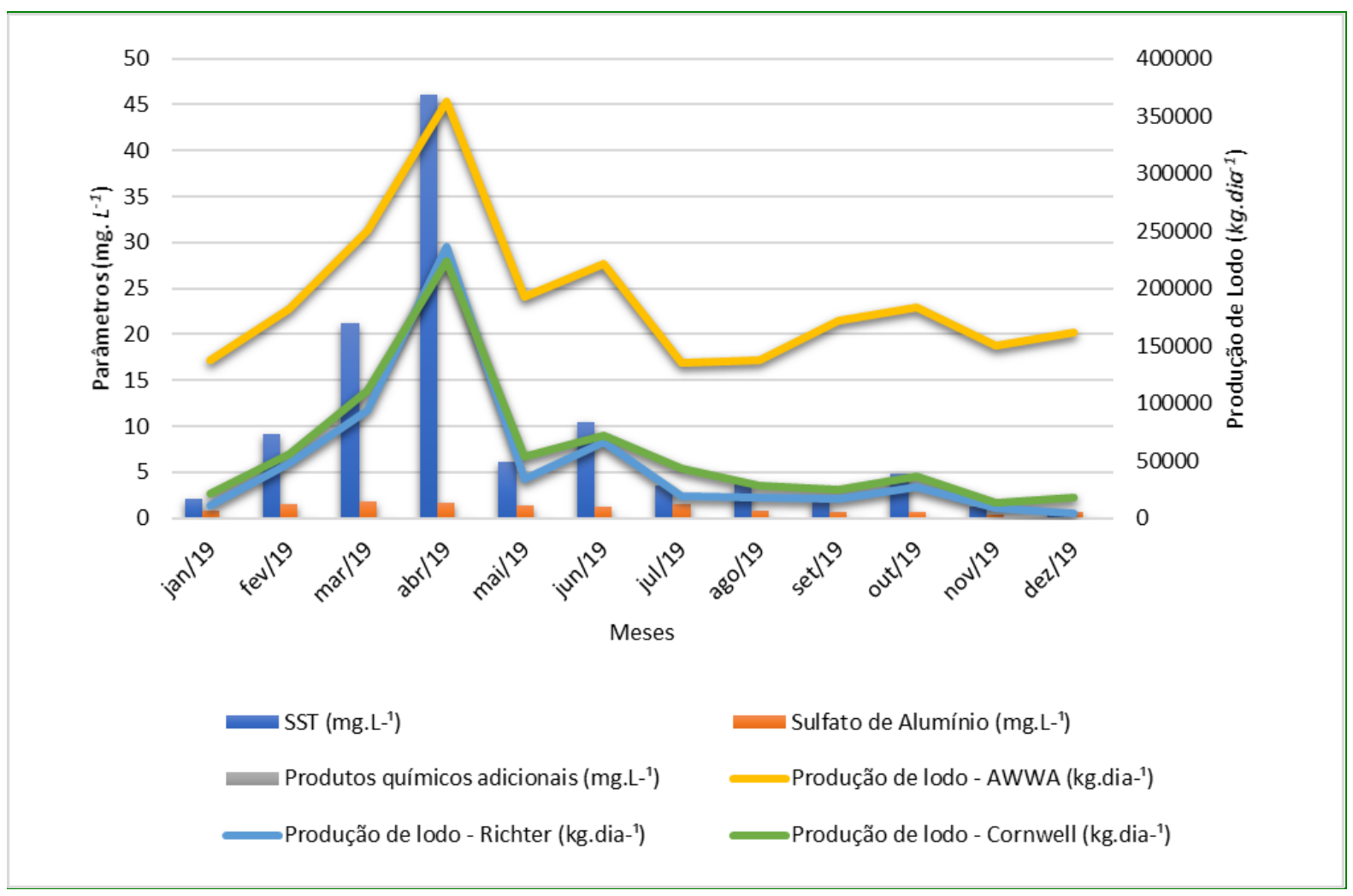

Fonte: Autores (2021).

Analisando os resultados da ETA João Ednaldo, percebe-se que a diferença entre os valores obtidos pelas equações propostas por Richter e por Cornwell é menor. Esse comportamento acontece devido ao fato de, nessa ETA, a quantidade de produtos químicos adicionados para o tratamento é menor em relação a ETA Poxim, se limitando, na maioria das vezes, apenas ao uso do coagulante, ainda que em quantidades menores. Já os valores da equação da AWWA se diferem das outras duas por envolver a dureza cálcica entre os parâmetros considerados.

As Tabelas 13 e 14 apresentam as estimativas mensais de produção de lodo para o ano de 2019 na ETA Poxim e João Ednaldo, respectivamente, e seus respectivos valores de precipitação média para região metropolitana de Aracaju encontrados por Ramos et al. (2020). Os valores de produção foram analisados por vazão de água tratada, a fim de não considerar este parâmetro e verificar a influência apenas das demais variáveis consideradas nas equações.

A produção de lodo na ETA Poxim teve seus picos em maio/junho e na ETA João Ednaldo, em abril, ainda dentro do período chuvoso conforme estudo de Ramos et al. (2020). Os valores de turbidez nesse mês, e nos adjacentes, culminaram em pico para 
Verificação da produção de lodo de eta em função da...

valores de sólidos suspensos totais calculados. Porém, os testes estatísticos mostraram que as correlações entre os valores de produção de lodo e a precipitação, para as equações estudadas em ambas as estações, são fracas ou moderadas, de acordo com convenção proposta por Callegari-Jacques (2003) apud Baba et al. (2014), que estabelece valores de 0,20 a 0,39 como correlações fracas e entre 0,40 e 0,69 como correlações moderadas.

Tabela 13 - Teste de Correlação ETA Poxim.

\begin{tabular}{ccccc}
\hline Meses & Precipitação & \multicolumn{3}{c}{ Produção de lodo (g.m $\mathbf{m}^{-3}$ ) em 2019 } \\
& Média (mm) & AWWA & RICHTER & CORNWELL \\
\hline Janeiro & 46,97 & 102,66 & 35,89 & 51,83 \\
Fevereiro & 63,94 & 98,34 & 20,04 & 51,76 \\
Março & 80,28 & 101,61 & 21,52 & 52,77 \\
Abril & 165,52 & 60,24 & 5,43 & 48,04 \\
Maio & 236,82 & 190,71 & 42,25 & 99,31 \\
Junho & 194,29 & 143,15 & 96,92 & 98,00 \\
Julho & 148,72 & 146,27 & 49,97 & 71,92 \\
Agosto & 97,62 & 119,16 & 44,56 & 65,94 \\
Setembro & 61,78 & 146,69 & 35,19 & 64,45 \\
Outubro & 57,00 & 130,09 & 62,52 & 84,30 \\
Novembro & 40,83 & 125,00 & 17,05 & 53,16 \\
Dezembro & 26,50 & 145,32 & 24,56 & 55,07 \\
Coeficiente de Correlação & $31 \%-$ FRACA & $38 \%-$ FRACA & $66 \%-$ MODERADA \\
\hline
\end{tabular}

Fonte: Autores (2021). 
Tabela 14 - Teste de Correlação ETA João Ednaldo.

\begin{tabular}{ccccc}
\hline Meses & Precipitação & \multicolumn{3}{c}{ Produção de lodo (g.m $\mathbf{m}^{-3}$ em 2019 } \\
& Média $(\mathbf{m m})$ & AWWA & RICHTER & CORNWELL \\
\hline Janeiro & 46,97 & 40,31 & 3,27 & 6,39 \\
Fevereiro & 63,94 & 55,05 & 14,51 & 16,74 \\
Março & 80,28 & 68,49 & 25,68 & 30,38 \\
Abril & 165,52 & 87,88 & 57,07 & 54,24 \\
Maio & 236,82 & 47,91 & 8,49 & 13,27 \\
Junho & 194,29 & 51,18 & 15,43 & 16,74 \\
Julho & 148,72 & 34,28 & 4,87 & 10,85 \\
Agosto & 97,62 & 38,30 & 4,99 & 7,74 \\
Setembro & 61,78 & 37,28 & 3,57 & 5,35 \\
Outubro & 57,00 & 42,21 & 6,16 & 8,25 \\
Novembro & 40,83 & 35,19 & 1,97 & 3,23 \\
Dezembro & 26,50 & 37,36 & 0,87 & 4,16 \\
Coeficiente de Correlação & $35 \%$ - FRACA & $38 \%-$ FRACA & $42 \%-$ MODERADA \\
\hline \multicolumn{5}{r}{} \\
\hline
\end{tabular}

É importante ressaltar que a relação entre sólidos em suspensão totais e a turbidez pode variar entre época de estiagem e de chuva, alterando valores de correlação. A medição do parâmetro SST representa melhor cenário para validar as relações para as ETAs em estudo. Segundo Katayama et al. (2015), parte do apelo prático das fórmulas empíricas é esvaziada ao se eliminar a perspectiva do uso de correlações entre SST e turbidez, sendo necessária a obtenção de amostragens para determinação de SST, as quais não foram possíveis para esta pesquisa.

\section{CONCLUSÕES}

Os resultados apresentados neste trabalho permitem observar que há variação entre os valores estimados pelas diferentes equações estudadas. Essa diferença foi menor quando foram aplicadas na estação onde a adição de produtos químicos é pequena, pois a variável que representa produtos adicionais passa a não influenciar muito em equações que consideram esse parâmetro. Assim, na ETA João Ednaldo, as equações de Richter e de Cornwell não apresentaram diferenças significativas.

Em ambas as ETAs, não houve, estatisticamente, aumento nem diminuição significativa de produção média de lodo ao longo dos anos de 2016 a 2019, em razão da semelhança entre a qualidade da água e a adição de produtos químicos. Porém, com base em estes estatísticos, foram observadas correlações fracas e moderadas, entre picos de 
produção de lodo entre meses do ano com registro de precipitação, tendo como influência o maior valor de sólidos suspensos em períodos chuvosos.

As variações entre os resultados mostram que a utilização de equações empíricas traz imprecisão, podendo causar incoerência com a realidade. Ainda assim, essas equações são úteis para estimativa de lodo, devido à maior viabilidade e à facilidade de cálculo, o que justifica a predominância no uso dessas equações.

Um estudo de probabilidade de acerto da estimativa com a realidade deve ser feito, comparando com a produção de lodo real, a fim de diminuir as diferenças entre o empírico e o exato. Para isso a companhia de saneamento deveria realizar medições de resíduos gerados durante o tratamento.

Conhecer características quantitativas e qualitativas da produção de lodo de uma ETA é necessário para poder ser realizado um esboço adequado e estratégico para o gerenciamento desse resíduo. O uso dos métodos empíricos é, portanto, uma alternativa praticável para se estimar e conhecer as características quantitativas. Deve-se, porém, conhecer a qualidade da água bruta e o que é utilizado no seu tratamento, para poder escolher o melhor método estimativo de produção de lodo.

Assim, uma ETA que utiliza produtos químicos em quantidade significativa, durante o tratamento, deve estimar a quantidade de produção de lodo por meio de equações que considerem maior quantidade de variáveis que representem os produtos adicionados, como as propostas pela AWWA e por Cornwell. No entanto, ETAs que não utilizam muitos produtos químicos (variedade e quantidade) podem optar por equações empíricas que desconsiderem essas variáveis, como a equação proposta por Richter.

\section{REFERÊNCIAS}

ASSOCIAÇÃO BRASILEIRA DE NORMAS TÉCNICAS - ABNT. NBR 10004: Resíduos sólidos - Classificação. 2004.

AHMAD, T.; AHMAD, K.; ALAM, M. Sludge quantification at water treatment plant and its management scenario. Environmental Monitoring and Assessment, v. 189, n. 9, 2017. DOI: 10.1007 / s10661-017-6166-1

AMERICAN WATER WORKS ASSOCIATION - AWWA. Water quality and treatment: a handbook of community water supplies. 5 ed. Ney York: McGraw-Hill, 1999. 
ARAGÃO, R.; SANTANA, G. R.; COSTA, C. E. F. F; CRUZ, M. A. S.; FIGUEIREDO, E. E.; SRINIVASAN, V. S. Chuvas intensas para o estado de Sergipe com base em dados desagregados de chuva diária. Revista Brasileira de Engenharia Agrícola e Ambiental, v. 17, n. 3, p. 243-252, 2013. DOI: 10.1590/S1415-43662013000300001

BABA, R. K.; VAZ, M. S. M. G.; COSTA, J. Correção de dados agrometeorológicos utilizando métodos estatísticos. Rev. Bras. Meteorol., São Paulo, Brasil, v. 29, n. 4, p. 515-526, 2014. https://doi.org/10.1590/0102-778620130611.

BARROSO, M. M.; CORDEIRO, J. S. Estudo de caso - ETA São Carlos (São Paulo/Brasil) produção de sólidos em ETAs, 2002, Cancún. Anais... Cancún: Congresso

Interamericano de Engenharia Sanitária e Ambiental, 2002. p. 1-9.

BATISTA, D. F.; MICHELAN, D. C. G. S.; BATISTA. I. F.; JESUS, V. A. Avaliação da eficiência da Estação de tratamento de água João Ednaldo/Sergipe, 2019, Aracaju.

Annals... Aracaju: XII Encontro de Recursos Hídricos em Sergipe, 2019.

BRASIL. Lei nำ12.305, de 2 de Agosto de 2010. Institui a Política Nacional de Resíduos Sólidos. Brasília, 2010.

BRASIL. Portaria de Consolidação $n^{\circ} 5$, de 28 de setembro 2017. Consolidação das normas sobre as ações e os serviços de saúde do Sistema Único de Saúde. Brasília, 2017.

CALLEGARI-JACQUES, S. M. Bioestatística: princípios e aplicações. Porto Alegre: Artemed, 255p, 2003.

CORNWELL, D. A. Handbook water treatment plant waste management. AWWA, Research Foundation, Denver, 431 p., 1987.

DESO. Distribuição de água. Disponível em: https://www.deso-

se.com.br/v2/index.php/agua/distribuicao-de-agua. Acesso em: 28.11.2020.

DI BERNARDO, L.; SABOGAL PAZ, L. P. Seleção de tecnologias de tratamento de água. v. 1 e 2. São Carlos: LDiBe. 1560 p., 2008.

FARIAS, M. C. V. Sub-bacia hidrográfica do rio Poxim: transformação da paisagem (1970-2010). 2011. 258f. Tese (Doutorado em Geografia) - Universidade Federal de Sergipe, São Cristóvão, 2011.

GOMES JUNIOR, G. R.; SOUZA, I. T. B. de; MENDONÇA, L. C.; ALMEIDA, J. O.; SANTOS, D. C. de G. Características dos lodos gerados em estação de tratamento de água (ETA), 2016, Florianópolis. Anais... Florianópolis: XVII SILUBESA - Simpósio LusoBrasileiro de Engenharia Sanitária e Ambiental. ABES, 2016.

HAUKOOS, J. S.; LEWIS, R. J. Advanced Statistics: Bootstrapping Confidence Intervals for Statistics with "Difficult" Distributions. Academic Emergency Medicine, v. 12, p. 360365, 2005. https://doi.org/10.1197/j.aem.2004.11.018

HOWE, K. J.; HAND, D. W.; CRITTENDEN J. C.; TRUSSELL, R. R.; TCHOBANOGLOUS, G. Princípios de Tratamento de Água. São Paulo: Cengage Learning. 620 p., 2017. 
INSTITUTO BRASILEIRO DE GEOGRAFIA E ESTATÍSTICA (IBGE). Pesquisa Nacional de Saneamento Básico (PNSB). Rio de Janeiro: Instituto Brasileiro de Geografia e Estatística. 2017.

KATAYAMA, V. T.; MONTES, C. P.; FERRAZ, T. H.; MORITA, D. M. Quantificação da produção de lodo de estações de tratamento de água de ciclo completo: Uma análise crítica. Engenharia Sanitária e Ambiental, v. 20, n. 4, p. 559-569, 2015. DOI: 10.1590/S1413-41522015020040105046

KAWAMURA, S. Integrat design of water treatment facilities, New York: John Wiley \& Sons, Inc. 720 p., 1991.

LIBÂNIO, M. Fundamento de qualidade e tratamento de água. 3. ed. Campinas, São Paulo: Editora Átomo, 494 p., 2010.

MARGUTI, A. L.; FERREIRA FILHO, S. S.; PIVELI, R. P. Full-scale effects of addition of sludge from water treatment stations into processes of sewage treatment by conventional activated sludge. Journal of Environmental Management, v. 215, p. 283-293, 2018. https://doi.org/10.1016/j.jenvman.2018.03.072

MOLINA, T. Caracterização e tratamento de água de lavagem de filtros de ETA com o uso de polímeros sintéticos e amido de batata. Revista de Engenharia e Tecnologia, v. 2, n. 3, p. 28-44, 2010.

MOREIRA, R. C. A.; GUIMARÃES, E. M.; BOAVENTURA, G. R. A; MOMESSO, A. M.; LIMA, G. L. Estudo geoquímico da disposição de lodo de estação de tratamento de água em área degrada. Química Nova, v. 32, n. 8, p. 2085-2093, 2009.

http://dx.doi.org/10.1590/S0100-40422009000800019

MOTTA SOBRINHO; M. A. DA; TAVARES, R. G.; ARRUDA, V. C. M.; CORREA, M. M.; PEREIRA, L. J. R. Geração, tratamento e disposição final dos resíduos das estações de tratamento de água do estado de Pernambuco. Engenharia Sanitária e Ambiental, v. 24, n. 4, p. 761-771, 2019. DOI: 10.1590/S1413-41522019175810

OLIVEIRA, L. C. de; PEREIRA, R.; VIEIRA, J. R. G. Análise da degradação ambiental da mata ciliar em um trecho do rio Maxaranguape - RN: uma contribuição à gestão dos recursos hídricos do Rio Grande do Norte - Brasil. Holos, ano 27, v. 15, 2011.

PRADO, J. R. S.; FIGEUIREDO, D. M.; DORES, E. F. G. C., HONGYU, K.; DELFINO, A. J. G.; RODRIGUES, D. V. B. Variação da qualidade da água em relação à sazonalidade e ao uso da terra no sul da região Amazônica. Caderno Prudentino de Geografia. V. 2, n. 43, p 159-184. 2021.

RAMIREZ, K. G.; POSSAN, E.; DEZEN, B. G.; COLOMBO, M. Potential uses of waste sludge in concrete production, Management of Environmental Quality, v. 28 n. 6, p. 821-838, 2017. https://doi.org/10.1108/MEQ-09-2015-0178

RAMOS, O. E. J. O.; MEDEIROS, E. S.; SILVA, A. Q.; OLIVEIRA, L. A.; BICALHO, C. C. Estudo do comportamento mensal das chuvas no Município de Aracaju, Estado do Sergipe, Brasil. Research, Society and Development, v. 9, n. 8, 2020. https://doi.org/10.33448/rsd-v9i8.6188 
REALI, M. A. P (Coordenador). Noções gerais de tratamento e disposição final de lodos de estações de tratamento de água, Rio de Janeiro - RJ, 225 p., 1999.

RICHTER, C. A. Água: métodos e tecnologias de tratamento. São Paulo: Blücher, 2009.

RICHTER, C. A. Tratamento de lodos de estações de tratamento de água. 1 ed. São Paulo: Blücher. 102 p., 2001.

ROCHA, R. M.; LUCAS, A. A. T.; ALMEIDA, C. A. P.; MENEZES NETO, E. L.; NETTO, A. O. A. Caracterização morfométrica da sub-bacia do rio Poxim-Açu, Sergipe, Brasil. Rev. Ambient. Água, v. 9, n. 2, p. 276-287, 2014. http://dx.doi.org/10.4136/ambi-agua.1289

SILVA, C. A.; SILVEIRA, C.; SILVA, F. A.; KLEN, M. R. F.; BERGAMASCO, R. Classificação dos lodos formados durante o processo de coagulação/floculação da água com os coagulantes PAC e Moringa Oleifera. Engevista, v. 14, n. 3. p. 302-309, 2012. https://doi.org/10.22409/engevista.v14i3.380

SUKSIRIPATTANAPONG, C.; HORPIBULSUK, S.; BOONGRASAN, S.; UDOMCHAI, A.; CHINKULKIJNIWAT, A.; ARULRAJAH, A. Unit weight, strength and microstructure of a water treatment sludge-fly ash lightweight cellular geopolymer, Construction and Building Materials, v. 94, p. 807-816, 2015. https://doi.org/10.1016/j.conbuildmat.2015.07.091

VESILIND, P. A.; MORGAN, S. M. Introdução à Engenharia Ambiental. Norteamericana: Cengage Learning, 456p., 2011. 


\begin{abstract}
Quantifying the sludge produced in water treatment plants (WTPs) is a fundamental step to know how much of this waste is improperly released into water sources and to plan its proper final disposal. This article discusses the comparison between three empirical methods for estimating the amount of sludge generated in two WTPs with different consumption of chemical products in water treatment for the municipality of Aracaju. The research was carried out in order to verify if there were differences in the results between the years 2016 to 2019, to compare the production estimates for each WTP and to analyze the influence of variations in the qualitative parameters of raw water and the addition of chemical products. It was found that empirical equations that consider a greater number of variables are the most appropriate for WTPs where treatment is more complex, while simpler equations can be suitable for WTPs that use few chemicals.
\end{abstract}

Keywords: Empirical equations. Sludge from WTP. Sludge quantification. Water treatment plant.

\title{
RESUMEN
}

La cuantificación de los lodos producidos en las Estacións de Tratamiento de Aguas (ETA) es un paso fundamental para saber qué cantidad de estos residuos se vierten indebidamente a las fuentes de agua y planificar su correcta disposición final. En este artículo se analiza la comparación entre tres métodos empíricos para estimar la cantidad de lodos generados en das ETA con diferente consumo de productos químicos en el tratamiento de aguas para el municipio de Aracaju. La investigación se realizó con el fin de verificar si existían diferencias en los resultados entre los años 2016 a 2019, comparar las estimaciones de producción para cada ETA y analizar la influencia de variaciones en los parámetros cualitativos del agua cruda y la adición de químicos. productos. Se encontró que las ecuaciones empíricas que consideran un mayor número de variables son las más apropiadas para las ETA donde el tratamiento es más complejo, mientras que las ecuaciones más simples pueden ser adecuadas para las ETA que utilizan pocos químicos.

Palabras-clave: Cuantificación de lodos. Ecuaciones empíricas. Estación de tratamiento de agua. Limo de ETA. 


\section{LICENÇA DE USO}

Este é um artigo publicado em acesso aberto (Open Access) sob a licença Creative Commons Atribuição 4.0 Internacional (CC BY 4.0), que permite uso, distribuição e reprodução em qualquer meio, desde que o trabalho original seja corretamente citado. Mais informações em: http://creativecommons.org/licenses/by/4.0

\section{CONFLITO DE INTERESSES}

Os autores declaram que não há conflito de interesses neste trabalho.

\section{CONTRIBUIÇÕES AUTORAIS}

Autor 1: Conceituação, curadoria de dados, investigação, escrita, revisão, edição, metodologia, primeira revisão, validação.

Autor 2: Conceituação, análise formal, investigação, administração, supervisão, escrita revisão, metodologia, segunda e terceira revisão, validação.

Autor 3: Conceituação, análise formal, investigação, administração, supervisão, escrita revisão, metodologia, segunda revisão, validação.

\section{FINANCIAMENTO}

O presente trabalho não contou com apoio financeiro.

\section{COMO REFERENCIAR}

SANTOS; Lucas Avelino Reis dos, MICHELAN; Denise Conceição de Gois Santos, JESUS; Taynar Mota de. Verificação da produção de lodo de ETA em função da quantidade e da qualidade da água bruta. Revista Brasileira de Engenharia de Biossistemas (Tupã), v. 15, n. 2, p. 235-258, 2021. DOI:

http://dx.doi.org/10.18011/bioeng2021v15n2p235-258.

\section{RESPONSABILIBADE EDITORIAL}

Prof. Dr. Fernando Ferrari Putti ${ }^{1}$, Prof. Dr. Paulo Sérgio Barbosa dos Santos ${ }^{1}$, Prof. Dr. Eduardo Festozo Vicente ${ }^{1}$ e Prof. Dr. Diogo de Lucca Sartori ${ }^{1}$

1 Universidade Estadual Paulista "Júlio de Mesquita Filho", FCE - Faculdade de Ciências e Engenharia, Tupã, SP, Brasil. 